Z Epileptol 2017 · 30:173

DOI 10.1007/s10309-017-0114-5

Online publiziert: 13. März 2017

๑) Springer Medizin Verlag GmbH 2017

CrossMark

\section{Leserbrief zu}

Trinka E et al (2016) Ergänzungen zur prächirurgischen Diagnostik bei Epilepsiepatienten. Z Epileptol. doi: 10.1007/s10309-016-0081-2

Mit Interesse und auch einer gewissen Verwunderung habe ich im letzten Heft der Zeitschrift für Epileptologie die Leserbriefdiskussion zu dem Artikel von Leonhardt et al. zur prächirurgischen Epilepsiediagnostik [1] verfolgt, insbesondere die Kritik der mangelnden Berücksichtigung der Magnetenzephalographie oder MEG [2-4]. Die beiden Gastherausgeber des entsprechenden Heftes haben in ihrem abschließenden Statement [5] meines Erachtens zutreffenderweise auch auf mögliche Interessenkonflikte der Leserbriefschreiber hingewiesen. Ich selbst habe während meiner Zeit als Medizinischer Direktor des Schweizerischen Epilepsie-Zentrums in Zürich auch einmal die Anschaffung eines MEG-Gerätes in Erwägung gezogen, mich nach ausführlicher Evaluation aber dagegen entschieden. Einer der Gründe war, dass mir einige langjährige Anwender der Methode (die die Geräte entweder von Herstellerfirmen kostenlos zur Verfügung gestellt bekommen oder im Rahmen von Forschungsprojekten finanziert hatten) versicherten, sich anderweitig entschieden zu haben, wenn sie die freie Wahl gehabt hätten, für den Anschaffungsbetrag andere Geräte zu kaufen.

Nun aber zu dem eigentlichen Anlass meines Leserbriefes. In einer der Stellungnahmen [3] heißt es im Zusammenhang mit Epilepsiemonitoring-Einheiten: „Dabei kann es nicht nur zu Verletzungen und Stürzen führen, sondern

Günter Krämer

Neurozentrum Bellevue, Zürich, Schweiz

\title{
Es gibt Boni und Mali, aber keine Stati (epileptici)
}

auch zu Stati epileptici oder periiktalen Psychosen“.

Obwohl ich kein „Lateiner“ bin (ich habe den naturwissenschaftlichen Zweig eines Gymnasiums gewählt, einen freiwilligen Lateinunterricht nach 4 Wochen frustriert abgebrochen und dann im Rahmen des Medizinstudiums einen obligaten Basiskurs belegen müssen), habe ich beim Lesen sofort ein ungutes Gefühl gehabt. Sowohl eine Rückfrage bei einem befreundeten Lateinlehrer als auch das Nachschlagen im Internet inklusive des Duden [6-8] haben bestätigt, dass es Status epileptici heißen muss. So sagt der Duden: „der Status; Genitiv: des Status, Plural: die Status [...tu:s]. Das aus dem Lateinischen stammende Substantiv lautet im Genitiv und im Plural gleich wie im Nominativ Singular, also des Status und die Status. Ein Unterschied besteht lediglich in der Aussprache. Im Plural wird das $u$ lang gesprochen." [6]

Eine andere Quelle [7] erläutert ausführlicher: „Das Lateinische kennt 7 Fälle (Casus, nicht etwa Casi), das sind 3 mehr als im Deutschen, und 5 Beugungen (also Deklinationen). Die Deklinationen werden von 1 bis 5 durchnummeriert, oder - je nach dem sog. Stammauslaut - z. B. auch als a-, o- oder u-Deklination bezeichnet. Die meisten maskulinen, also männlichen Substantive gehören entweder zur 2. Deklination = o-Deklination oder zur 4. Deklination = u-Deklination. Kategorien der Deklination sind das Geschlecht (männlich = Maskulinum, weiblich = Femininum, sächlich = Neutrum), die Zahl (Einzahl = Singular, Mehrzahl = Plural) und der Fall (Nominativ = WerFall, Genitiv = Wes-Fall, Dativ = WemFall, Akkusativ = Wen-Fall, und im Lateinischen noch Ablativ - von Schülern scherzhaft als ,Durch-Fall` bezeichnet,
Lokativ $=$ Wo-Fall und Vokativ $=$ Anrede). Substantive der o-Deklination bilden den Plural mit -i (wie z. B. dominus, domini - der Herr, die Herren oder populus, populi - das Volk, die Völker). Substantive der u-Deklination dagegen bilden den Plural mit -us (wie z. B. portus, portus - der Hafen, die Häfen oder casus, casus - der Fall, die Fälle oder eben unser Untersuchungsobjekt ,Status').“

Vielleicht tröstet es die Sprachverwirrer, dass sie nicht alleine sind. Auf dem Rechtschreibeportal „korrekturen.de“ haben „die Stati“ Eingang in die Sammlung beliebter Fehler gefunden [8].

\section{Korrespondenzadresse}

Dr. med. G. Krämer

Neurozentrum Bellevue

Theaterstr. 8, 8001 Zürich, Schweiz

g.kraemer@epilepsie-med.de

Interessenkonflikt. G. Krämer gibt an, dass kein Interessenkonflikt besteht.

\section{Literatur}

1. Leonhardt G (2016) Aktuelle Prächirurgische Diagnostik. ZEpileptol 29:106-114

2. Kasper BS, Rampp S, Hamer HM, Graf W, Pauli E, Schwarz M, Rössler K, Sommer B, Stefan H (2017) Prächirurgische Epilepsiediagnostik. WADA-Test und MEG sind wertvoll. ZEpileptol 30:71-72

3. Trinka E, Kreidenhuber R, Leitinger M (2017) Ergänzungen zur prächirurgischen Diagnostik bei Epilepsiepatienten.ZEpileptol 30:73-74

4. Boon P (2017) Kommentar zu G. Leonhardts „Presurgical evaluation - state of the art" (2016) im Auftrag der European MEG Society (EMEGS). ZEpileptol 30:75

5. Schulze-Bonhage A, Steinhoff BJ (2017) MEG als Element der Epilepsiediagnostik? Z Epileptol 30:77-78

6. http://www.duden.de/rechtschreibung/Status. Zugegriffen: 17.02.2017

7. http://status-mehrzahl.de/. Zugegriffen: 17.02.2017

8. https://www.korrekturen.de/beliebte_fehler/ stati.shtml.Zugegriffen: 17.02.2017 